\title{
Fogitare
}

\section{NO EPICENTRO DA EPIDEMIA: UM OLHAR SOBRE A COVID-19 NA ITÁLIA}

Elisabetta Riboli', Juliana Perez Arthur², Maria de Fátima Mantovani ${ }^{3}$

\section{RESUMO}

Objetivo: refletir sobre os impactos da COVID-19 no norte da Itália, epicentro europeu da pandemia. Desenvolvimento: a abordagem desenvolvida foi a partir do surgimento da doença, sua chegada na Itália e suas repercussões, não apenas no âmbito sanitário, como também nos relacionamentos humanos e no ambiente social. A epidemia vem impactando a vida dos profissionais da saúde e mobilizou avanços em pesquisas em todo o mundo.

Considerações finais: considera-se que o enfrentamento da doença no país evidenciou o protagonismo da enfermagem como profissão do cuidar e permitirá um novo pensar sobre o ensino, a assistência e a pesquisa da enfermagem.

DESCRITORES: Enfermagem; Pandemias; Infecções por Coronavírus; Cuidados de Enfermagem.

COMO REFERENCIAR ESTE ARTIGO:

Riboli E, Arthur JP, Mantovani M de F. No epicentro da epidemia: um olhar sobre a Covid-19 na Itália. Cogitare enferm. [Internet]. 2020 [acesso em "colocar data de acesso, dia, mês abreviado e ano"]; 25. Disponível em: http://dx.doi.org/10.5380/ce.v25i0.72955.

Este obra está licenciado com uma Licença Creative Commons Atribuição 4.0 Internacional.

${ }^{1}$ Enfermeira. Especialista em Enfermagem comunitária. Ospedale Maggiore di Crema. Crema, Lombardia, Italia. $(8$ ${ }^{2}$ Enfermeira. Mestre em Enfermagem. Especialista em Enfermagem comunitária. Milão, Lombardia, Italia. (]

${ }^{3}$ Enfermeira. Doutora em Enfermagem. Docente de Pós-Graduação em Enfermagem da Universidade Federal do Paraná.

Curitiba, PR, Brasil. 


\title{
IN THE EPICENTER OF THE EPIDEMIC: A LOOK AT COVID-19 IN ITALY
}

\begin{abstract}
Objective: To reflect on the impacts of COVID-19 in northern Italy, the European epicenter of the pandemic.

Development: The approach developed since the advent of the disease, its arrival in Italy and its repercussions, not only in the health field, but also in the human relationships and the social environment. The epidemic has been impacting the lives of health professionals and has mobilized advances in research around the world.

Final considerations: It is considered that coping with the disease in the country evidenced the role of Nursing as a care profession and that it will allow for a new thinking about Nursing teaching, care, and research.
\end{abstract}

DESCRIPTORS: Nursing; Pandemics; Infections by Coronavirus; Nursing Care.

\section{EN EL EPICENTRO DE LA EPIDEMIA: UNA MIRADA AL COVID-19 EN ITALIA}

\begin{abstract}
RESUMEN:
Objetivo: reflexionar sobre los efectos del COVID-19 en el norte de Italia, el epicentro europeo de la pandemia.

Desarrollo: el enfoque que se desarrolló se basó en la aparición de la enfermedad, su llegada a Italia y sus repercusiones, no solo en el ámbito sanitario sino también en las relaciones humanas y en el ambiente social. La epidemia ha afectado la vida de los profesionales de la salud y ha movilizado avance en diversas investigaciones en todo el mundo.

Consideraciones finales: se considera que la lucha contra la enfermedad en este país evidenció el protagonismo de la Enfermería como profesión dedicada a prestar cuidados, y permitirá elaborar una nueva manera de pensar en lo que respecta a la enseñanza, la asistencia y la investigación en Enfermería.
\end{abstract}

DESCRIPTORES: Enfermería; Pandemias; Infecciones por Coronavirus; Cuidados de Enfermería. 
Era dezembro de 2019 quando um vírus, até então desconhecido, chamado de SARSCoV-2 (Severe acute respiratory syndrome coronavirus 2), surge na China. Acompanhando de longe os noticiários, todos pensávamos que, assim como já aconteceu com outros vírus, tudo não passaria de casos isolados ou ao máximo desencadearia uma endemia que seria rapidamente controlada, mas não foi o que aconteceu.

A epidemia por esse vírus eclodiu em Wuhan, uma cidade importante na China, altamente densa com uma população de mais de 14 milhões, em 2019. Os estudos iniciais demonstravam que o vírus era altamente contagioso, mais que os outros subtipos de Corona vírus responsáveis pelas Síndromes Respiratórias Agudas Graves (SARS) e Síndrome Respiratória do Oriente Médio (MERS), porém com menor taxa de mortalidade ${ }^{(1)}$.

$\mathrm{Na}$ Itália, onde vivo atualmente, no começo do ano de 2020 se ouvia falar sobre a doença, mas as fronteiras com o Oriente pareciam criar uma espécie de falsa proteção. Entretanto, rapidamente vimos a situação se agravar: cidades inteiras na China em isolamento domiciliar, comércios fechados e somente os serviços essenciais funcionando. Quando menos esperávamos, ele chegou ao Norte da Itália, e veio com intensidade, transformando a vida cotidiana em caos.

A origem do vírus, seus hospedeiros e mecanismos de transmissão para o homem foram objetos de atenção em todo o mundo. Um estudo realizado em Hong Kong apontou que se trata, provavelmente, de um novo vírus recombinante, cujo genoma é próximo ao Corona vírus relacionados à Síndrome Respiratória Aguda Grave, de morcegos-ferradura e que, embora inicialmente se suspeitasse que o mercado de Wuhan fosse o epicentro da epidemia, a fonte imediata ainda permanece desconhecida ${ }^{(2)}$.

A doença causada pelo SARS-CoV2 foi denominada pela Organização Mundial de Saúde (OMS) de COVID-19(3). Sabe-se que as pessoas contaminadas podem apresentar alguns sintomas, incluindo febre, congestão nasal, dificuldade respiratória, tosse, e lesões invasivas nos pulmões ${ }^{(4)}$. Muitos dos infectados não apresentam sintomas e se recuperam sem a necessidade de um tratamento específico. Entretanto, cerca de uma em cada seis pessoas acometidas pela COVID-19 fica gravemente doente, sendo os idosos e pessoas com comorbidades associadas os mais afetados ${ }^{(5)}$.

Um dos desafios relacionados à doença é o fato de o vírus ser transmissível tanto durante o período de incubação quanto na infecção recessiva ${ }^{(1)}$. Dados da OMS apontam que, até dia 29 de abril de 2020, o número de países atingidos pelo SARS-CoV2 era 213, as pessoas diagnosticadas passavam de 3 milhões e os óbitos somavam 207.973 em todo o mundo(6).

\section{A CHEGADA DO COVID-19 NA ITÁLIA}

Os dois primeiros casos de COVID-19 na Itália foram de um casal de turistas chineses, que no dia 30 de janeiro tiveram a confirmação da doença no Instituto Spallanzani, em Roma, onde foram isolados ${ }^{(7)}$. $O$ primeiro caso de transmissão secundária ocorreu em Codogno, região da Lombardia, norte do país, no dia 18 de fevereiro de 2020.

O crescimento dos casos no país foi assustador. Uma semana após o caso um de transmissão secundária ser confirmado (25 de fevereiro de 2020), o número de diagnosticados por COVID-19 na Itália era de 323 e um mês depois (18 de março de 2020), já eram $35.713^{(8)}$.

No começo de março de 2020, o governo italiano emitiu uma série de decretos, 
incialmente para as regiões mais afetadas pela doença e posteriormente válidas para todo o território nacional, para o controle e contenção da COVID-19. Houve proibição de agrupamento de pessoas em lugares abertos e fechados, suspensão de eventos esportivos, fechamento de serviços que não fossem de primeira necessidade, etc ${ }^{(9)}$, dando início a um isolamento para boa parte da população. Próximo à segunda quinzena de março de 2020, a OMS, frente ao crescente número de pessoas contagiadas, óbitos e países com casos confirmados, anunciou que o surto de COVID-19 era uma pandemia(10).

Dados do Istituto Superiore di Sanità italiano apontam que, no dia 16 de abril, o número total de pessoas que tiveram um exame que confirmava o contágio pelo novo vírus na Itália era de 159.107, o número de óbitos relacionado à doença era de 19.996 e o número de pessoas curadas $40.164^{(8)}$.

A média de idade dos pacientes que foram a óbito era de 79 anos, mais de 15 anos superior à dos pacientes que contraíram a infecção, cuja idade média é de 62 anos. As pessoas do sexo masculino (66\%) eram as mais acometidas, assim como aquelas com patologias associadas. Dos pacientes que faleceram, 61,5\% tinham três ou mais patologias no momento da internação, 20,7\% apresentavam duas patologias preexistentes, 14,5\% possuíam uma e 3,3\% nenhuma patologia(11).

A Itália é um país característico pelo grande número de pessoas idosas. Dados do Istituto Nazionale di Statistica (Istat) demonstram um constante crescimento em termos absolutos e relativos da população idosa no país; em janeiro de 2019 a população acima de 65 anos representava 22,8\% do total, enquanto os jovens até 14 anos eram cerca de $13,2 \%{ }^{(12)}$. Considerando que a COVID-19 pode ser especialmente perigosa nesta faixa etária, as características da população podem ter contribuído para o número de óbitos.

A chegada da doença na Itália foi inesperada, mas, principalmente, colapsou um sistema sanitário de excelência, que se viu despreparado frente a um alto número de internamentos por síndrome respiratória. Certamente, para os profissionais de saúde, no início era tudo mais difícil, mais dramático. Diante dos primeiros casos, os hospitais se prepararam para acolher os pacientes, mas não estavam prontos para o grande número de pessoas que chegou dias depois. Os enfermeiros, na linha de frente, viram suas rotinas mudarem completamente, e tentavam dar o melhor de si, mesmo com desgaste físico e principalmente o desgaste emocional. A angústia do desconhecido era fácil de ser notada por trás das máscaras e um dos maiores medos dos profissionais não era se contagiar, mas sim de levar para casa a doença e contagiar um ente querido.

Diante da questão da transmissão do vírus aos profissionais de saúde, um dos artigos pioneiros sobre o novo Coronavírus publicado por chineses aponta que, no dia 11 de fevereiro de 2020, 1.716 profissionais da saúde tinham sido infectados e seis deles morreram na China. Além disso, quando os sintomas dessas pessoas não são óbvios e fáceis de serem identificados, as famílias dos profissionais correm alto risco de infecção(1).

Infelizmente, o número de profissionais de saúde e de familiares infectados continuou crescendo, na China e no mundo. Na Itália, até o dia 16 de abril, foram diagnosticados 16.991 casos em profissionais da saúde, representando 10,7\% do total de casos relatados no país. A mediana de idade desses profissionais era de 48 anos e $68 \%$ eram do sexo feminino ${ }^{(8)}$.

O dia a dia dos enfermeiros italianos nos hospitais com maior fluxo de COVID-19 positivos, sem dúvida, não era fácil. A notícia de que um colega tinha sido infectado ou tinha ido a óbito sobrecarregava os ombros já cansados. Por outro lado, depoimentos de enfermeiros que trabalham na linha de frente relatam que, no meio do caos, era difícil ver um paciente que se lamentava, pelo contrário, eles se ajudavam. Não eram raros os momentos em que pacientes jovens ajudavam os mais velhos na alimentação e higiene, ou pessoas de mais idade que davam preferência aos jovens na realização de exames e demais procedimentos. Eram esses os momentos que davam esperança de dias melhores. Os jornais retratavam os profissionais da saúde como heróis, mas não era assim que eles se sentiam; para eles, os verdadeiros heróis eram os próprios pacientes. 
A emergência Coronavírus ativou alarmes em relação à saúde mental dos trabalhadores. Um estudo realizado com 1.257 profissionais da saúde chineses, entre enfermeiros e médicos, mostrou que muitos profissionais da área relataram sintomas depressivos, ansiedade, insônia e angústia. Estes sintomas eram principalmente em mulheres enfermeiras em Wuhan e demais profissionais que se dedicam diretamente ao diagnóstico, tratamento ou prestação de cuidados a pacientes suspeitos ou COVID-19 confirmados ${ }^{(13)}$.

Na Itália, com o tempo e as medidas de combate adotadas, os casos foram diminuindo, os hospitais foram se organizando e a queda no número de casos deu um fôlego a mais a todos. O medo ainda persiste e algumas considerações sobre essa nova pandemia já podem ser feitas.

Ainda que já tivesse se alastrado de maneira impactante no país mais populoso do mundo, o novo Coronavírus chegou ao Ocidente de maneira silenciosa. Um estudo que utilizou dados de viagens aéreas sugere que aproximadamente dois terços dos casos de COVID-19 exportados da China não foram detectados ${ }^{(14)}$.

Em um mundo globalizado, com um grande fluxo quotidiano de pessoas entre países, era esperado que a doença se difundisse. Entretanto, cada país acometido pela doença enfrentou a batalha com surpresa, alguns com dados mais dramáticos que outros, porém sempre com muito medo e insegurança.

De fato, a sensação que temos é que nenhum país estava realmente preparado para o enfrentamento dessa doença. A precursora da enfermagem moderna, Florence Nightingale, organizou suas teorias em um momento de guerra, mas com o passar dos anos e o avanço da medicina, a preparação para momentos de emergência perdeu o protagonismo e ficou em segundo plano. A formação do enfermeiro, tanto no Brasil como na Itália, frequentemente não aborda disciplinas que nos instrumentalizem para momentos tão delicados como esse. As grandes epidemias são estudadas a título de curiosidade, mas a ideia de que uma pandemia possa acontecer parece, ou parecia, muito improvável.

Nesse momento, é necessária uma cooperação global para vencer a guerra pandêmica. Cientistas estão trabalhando para entender a COVID-19 e ações abrangentes em todo o mundo são importantes para avançar em direção à solução(1). A doença trouxe à luz uma série de fragilidades desconhecidas, evidenciou a necessidade de mudanças no sistema sanitário, a carência de profissionais de saúde e a importância do investimento na ciência. É o assunto de 24 horas por dia nos jornais locais e o assunto recorrente entre amigos e familiares.

O isolamento social na Itália obrigou as pessoas a mudarem hábitos de vida, de alimentação, lazer e trabalho. A vida social passou a acontecer pelas janelas e sacadas das casas e apartamentos. Os quintais ganharam um valor especial e as redes sociais serviam de ponte entre amigos, familiares e amores, separados fisicamente. A pandemia fez com que as pessoas se sentissem um pouco mais responsáveis umas pelas outras. Floresceram as relações entre vizinhos, mas, sobretudo, deixou marcas profundas naqueles que sentiram de perto o impacto da perda de um ente querido e naqueles trabalhadores essenciais que carregavam nas costas o peso dessa batalha.

Hoje, a doença ainda está se espalhando pelo mundo, causando mortes prematuras e levando embora a base de tantas famílias, que são os idosos. O mundo espera com ânsia o desenvolvimento de uma vacina e de remédios verdadeiramente eficazes. Enquanto isso, nos adaptamos e aprendemos a conviver.

\section{CONSIDERAÇÕES FINAIS}

A experiência da Itália como primeiro epicentro ocidental de SARS-CoV-2 está sendo 
intensa e mobilizando o mundo a avanços em pesquisas clínicas e no âmbito da saúde em todo o mundo. Juntamente a isso, a pandemia evidenciou um real protagonismo da enfermagem, cuja expertise é o cuidar.

Podemos observar que as vivências com o combate à doença nos diversos países acometidos, ainda que com suas particularidades, foram semelhantes em muitos aspectos. Neste sentido, a troca de experiências entre enfermeiros que estiveram, e ainda estão, na linha de frente de combate, poderia resultar em diretrizes de atendimento às pessoas com COVID-19, que evidenciem as melhores práticas universais de cuidados, materiais e equipamentos adequados e que permitam a qualidade do atendimento e a segurança do profissional.

Por fim, o que se espera é que as mortes ocasionadas por essa nova doença não tenham sido em vão, que os países consigam se reestruturar econômica e socialmente e que essa experiência sirva como contribuição para um novo pensar da enfermagem, das fragilidades da sociedade e valorização da ciência.

\section{REFERÊNCIAS}

1. Zhu H, Wei L, Niu P. The novel coronavirus outbreak in Wuhan, China. Glob health res policy. [Internet]. 2020 [acesso em 21 abr 2020]; 5(6). Disponível em: https://doi.org/10.1186/s41256-020-00135-6.

2. Lau SKP, Luk HKH, Wong ACP, Li KSM, Zhu L, He Z, et al. Possible bat origin of severe acute respiratory syndrome Coronavirus 2. Emerg Infect Dis. [Internet]. 2020 [acesso em 22 abr 2020]; 26(7). Disponível em: https://doi.org/10.3201/eid2607.200092.

3. World Health Organization (WHO). Naming the coronavirus disease (COVID-19) and the virus that causes it. [Internet]. 2020 [acesso em 21 abr 2020] Disponível em: https://www.who.int/emergencies/ diseases/novel-coronavirus-2019/technical-guidance/naming-the-coronavirus-disease-(covid-2019)-andthe-virus-that-causes-it.

4. Song F, Shi N, Shan F, Zhang Z, Shen J, Lu H, et al. Emerging 2019 Novel Coronavirus (2019-nCoV) Pneumonia. Radiology. [Internet]. 2020 [acesso em 14 abr 2020]; 295(1). Disponível em: https://doi. org/10.1148/radiol.2020200274.

5. World Health Organization (WHO). Q\&A on coronaviruses (COVID-19). [Internet]. 2020 [acesso em 21 abr 2020]. Disponível em: https://www.who.int/news-room/q-a-detail/q-a-coronaviruses.

6. World Health Organization (WHO). Coronavirus disease 2019 (COVID-19) situation report - 100 [Internet]. 2020 [acesso em 30 abr 2020]. Disponível em: https://www.who.int/docs/default-source/ coronaviruse/situation-reports/20200429-sitrep-100-covid-19.pdf?sfvrsn=bbfbf3d1 6 .

7. Ministero della Salute (ITA). Nuevo coronavirus. News e media - Notizie. Nuovo Coronavirus, consiglio dei ministri dichiara stato d'emergenza. [Internet]. 2020 [acesso em 21 abr 2020]. Disponível em: http://www.salute.gov.it/portale/nuovocoronavirus/dettaglioNotizieNuovoCoronavirus. jsp? lingua=italiano\&menu=notizie \&p=dalministero\&id $=4035$.

8. Istituto Superiore di Sanità. Task force COVID-19 del Dipartimento Malattie Infettive e Servizio di Informatica, Istituto Superiore di Sanità. Epidemia COVID-19, aggiornamento nazionale: 16 aprile 2020. [Internet]. 2020 [acesso em 17 abr 2020]; Disponível em: https://www.epicentro.iss.it/coronavirus/ bollettino/Bollettino-sorveglianza-integrata-COVID-19 16 -aprile-2020.pdf.

9. Italia. Decreto del Presidente del Consiglio dei Ministri. Ulteriori disposizioni attuative del decreto-legge 23 febbraio 2020, n. 6, recante misure urgenti in materia di contenimento e gestione dell'emergenza epidemiologica da COVID-19, applicabili sull'intero territorio nazionale. Gazzeta Ufficiale della Reppublica Italiana, 9 mar 2020; Serie generale n 62.

10. World Health Organization (WHO). Director-General's opening remarks at the media briefing on 
COVID-19 - 11 March 2020. [Internet]. 2020 [acesso em 30 mar 2020]. Disponível em: https://www.who. $\mathrm{int} / \mathrm{dg} / \mathrm{speeches/detail/who-director-general-s-opening-remarks-at-the-media-briefing-on-covid-19---11-}$ march-2020.

11. Istituto Superiore di Sanità. Gruppo della Sorveglianza COVID-19. Caratteristiche dei pazienti deceduti positivi all'infezione da SARS-CoV-2 in Italia Dati al 16 aprile 2020. [Internet]. 2020 [acesso em 17 abr 2020]; Disponível em: https://www.epicentro.iss.it/coronavirus/bollettino/Report-COVID-2019 16 aprile.pdf.

12. Istituto Nazionale di Statistica (Istat). Statistiche Report. Indicatori Demografici. [Internet]. 2019 [acesso em 17 abr 2020]; Disponível em: https://www.istat.it/it/files/2019/02/Report-Stime-indicatoridemografici.pdf.

13. Lai J, Ma S, Wang Y, Cai Z, Hu J, Wei N, et al. Factors associated with mental health outcomes among health care workers exposed to Coronavirus disease 2019. JAMA Netw Open. [Internet]. 2020 [acesso em 12 abr 2020]; 3(3). Disponível em: http://doi.org/10.1001/jamanetworkopen.2020.3976.

14. Bhatia S, Imai N, Cuomo-Dannenburg G, Baguelin M, Boonyasiri A, Cori A, et al. Report 6: relative sensitivity of international surveillance. Imperial College London. [Internet]. 2020 [acesso em 12 abr 2020]; Disponível em: https://doi.org/10.25561/77168.

Recebido: $17 / 04 / 2020$

Finalizado: 11/05/2020

Editora associada: Luciana Puchalski Kalinke

Autor Correspondente:

Juliana Perez Arthur

Universidade Federal do Paraná

Av. Prefeito Lothário Meissner, 632 - 80210-170 - Curitiba, PR, Brasil

E-mail: julianaperez.4@gmail.com

Contribuição dos autores:

Contribuições substanciais para a concepção ou desenho do estudo; ou a aquisição, análise ou interpretação de dados do estudo - ER, JPA

Elaboração e revisão crítica do conteúdo intelectual do estudo - JPA, MFM

Aprovação da versão final do estudo a ser publicado - ER, JPA, MFM

Responsável por todos os aspectos do estudo, assegurando as questões de precisão ou integridade de qualquer parte do estudo - JPA 